\title{
La contestation transnationale
}

\section{Sidney Tarrow}

\section{(2) OpenEdition}

\section{Journals}

\section{Édition électronique}

URL : http://journals.openedition.org/conflits/276

DOI : 10.4000/conflits.276

ISSN : 1777-5345

Éditeur :

CCLS - Centre d'études sur les conflits lilberté et sécurité, L'Harmattan

\section{Édition imprimée}

Date de publication : 1 juin 2000

ISBN : 2-7384-9946-5

ISSN : 1157-996X

\section{Référence électronique}

Sidney Tarrow, «La contestation transnationale », Cultures \& Conflits [En ligne], 38-39 | été-automne

2000, mis en ligne le 20 mars 2006, consulté le 30 mars 2021. URL : http://journals.openedition.org/ conflits/276 ; DOl : https://doi.org/10.4000/conflits.276

Ce document a été généré automatiquement le 30 mars 2021.

Creative Commons License 


\title{
La contestation transnationale
}

\author{
Sidney Tarrow
}

1 «Au cours de deux siècles d'exception » déclare Charles Tilly, « les pays européens et leurs extensions sur les autres continents ont remarquablement réussi à circonscrire et à contrôler les ressources se trouvant à l'intérieur de leurs frontières... Mais à notre époque... au moins en Europe, l'ère des Etats forts tire maintenant à sa fin ${ }^{1}{ }^{1}$. Tilly admet volontiers que sa déclaration se fonde sur une "série de spéculations, de conjectures et d'hypothèses ». Mais admettons, au moins pour le moment, la justesse de cette intuition et la réalité du déclin de l'Etat fort et consolidé sur le modèle westphalien. La question pour ceux qui étudient la politique de la contestation et les relations internationales est de savoir si ce déclin a) est cyclique et sera compensé par la remarquable capacité d'adaptation et de renouvellement des Etats; b) est en train d'être remplacé par des formes de gouvernance institutionnelle non-territoriale; c) donne l'opportunité à des mouvements sociaux et d'autres formes nongouvernementales d'action collective de venir occuper un espace politique normalement dévolu aux institutions; ou d) donne lieu à une combinaison de ces trois facteurs. Certains chercheurs résolvent cette énigme en prédisant une montée en puissance de nouveaux organismes de gouvernance internationale ${ }^{2}$, tandis que d'autres prévoient des mouvements sociaux nationaux dépassant les frontières pour créer une sorte de «société civile mondiale ${ }^{3}$ D'autres encore envisagent des «réseaux transnationaux de militants" reliant de nouvelles formes de gouvernance aux anciennes et représentant les intérêts d'acteurs pauvres en ressources à l'intérieur des Etats ${ }^{4}$ alors que certains penchent pour une combinaison d'acteurs gouvernementaux et non-gouvernementaux, nationaux et internationaux ${ }^{5}$. Notre idée force est que la plupart de ces prévisions, quoique fondées sur des preuves solides, ne procèdent pas d'une vision d'ensemble et ne parviennent pas à prendre en compte les interactions entre les mouvements sociaux, les organisations non-gouvernementales, les Etats et les institutions internationales. Les auteurs de ces hypothèses proposent, en particulier, fort peu de mécanismes reliant les acteurs nationaux aux acteurs transnationaux et aux institutions internationales. Mon propos est de défendre l'idée que les institutions internationales, créées par les Etats pour servir des intérêts communs, ont un effet d'ancrage et de montée en puissance pour les acteurs non-étatiques, et leur fournissent 
les ressources, les opportunités et la motivation grâce auxquelles ils peuvent s'organiser et se mobiliser à l'échelon transnational. Au lieu d'être perçues comme étant aux antipodes de la contestation transnationale, les institutions internationales peuvent offrir ressources, opportunités et motivation au militantisme transnational. Trois mises en garde Avant d'aborder ces questions, il serait bon de nous rappeler trois leçons que nous a données l'histoire, et qu'oublient trop souvent les augures d'une société civile mondiale à brève échéance : Les Etats restent forts dans la majorité des domaines décisionnels - par exemple dans le maintien de la sécurité nationale - même si leur capacité de contrôle des flux de capitaux s'est affaiblie'. Ils gardent le contrôle de leurs frontières et conservent leur souveraineté légale sur leur territoire. Leurs ressortissants peuvent voyager plus facilement que par le passé, ils peuvent constituer des réseaux dépassant le cadre de ces frontières ${ }^{7}$ mais ils vivent encore dans des pays et peuvent user, au moins dans ceux qui sont démocratiques, des opportunités, des réseaux et du répertoire bien connu des moyens d'action disponibles de leur système politique national ${ }^{8}$. Il s'agit là de ressources que les hypothétiques sirènes de la "société civile mondiale» peuvent difficilement concurrencer. La solidarité transnationale, dont on impute fréquemment l'origine à la "mondialisation » économique ${ }^{9}$, n'en existe pas moins depuis un siècle au bas mot, et même bien davantage si l'on prend en compte la révolution «atlantique » du XVIIIe siècle ou la Réforme protestante ${ }^{10}$. Il ne s'agit pas là de simples arguties historiques. L'apparition historiquement bien antérieure à la «mondialisation" des organisations transnationales implique qu'il nous faudra spécifier des mécanismes responsables du développement actuel de l'organisation et de la contestation transnationales autres qu'économiques Les mouvements sociaux, les réseaux transnationaux et les ONG ne sont pas les seuls acteurs à opérer de façon transnationale. Les Etats ont toujours dépassé le cadre de leurs frontières ${ }^{11}$. C'est une tendance qui s'accroît à l'heure actuelle, notamment par la signature d'accords internationaux, l'intervention dans les affaires intérieures d'autres pays (généralement plus faibles) et la création d'institutions internationales. Ces institutions dirigées au niveau des Etats répondent habituellement à des buts étatiques ${ }^{12}$, souvent dans le dessein de contrecarrer des activités transnationales que les Etats ne peuvent contrôler ${ }^{13}$ ou de fournir «l'assurance » que d'autres Etats ne faillissent pas à leurs engagements ${ }^{14}$. Les Etats dominants du système international exercent en outre une puissante influence sur les relations transnationales, non seulement en contrôlant les acteurs non-étatiques mais aussi en fournissant des modèles de politique transnationale à partir de leurs propres systèmes nationaux ${ }^{15}$. 薄 Le militantisme transnational ayant diverses sources, dont certaines réversibles, il existe des variations dans le degré de "transnationalisation» des différents secteurs et il se peut qu'il n'existe pas de tendance unidirectionnelle vers une société civile mondiale, même si l'inverse semble être le cas dans le moment historique où nous nous trouvons actuellement. Je commencerai cet article par une rapide étude des changements dans la façon dont les études américaines de relations internationales traitent des activités politiques transnationales depuis les années soixante-dix. Ma seconde partie dégagera les contributions d'un nouveau groupe de chercheurs, spécialisés dans la politique de la contestation, à ce champ de recherche. Ma troisième partie distinguera trois types principaux d'acteurs transnationaux, les mouvements sociaux transnationaux, les organisations internationales non-gouvernementales (OING), et les réseaux de militants transnationaux. Ma quatrième partie se consacrera aux relations hypothétiques entre la contestation transnationale et les institutions 
internationales. En conclusion, j'évoquerai les contours possibles d'une société politique européenne. Des anciennes aux nouvelles relations transnationales ${ }^{16}$ Le paradigme sous l'angle duquel les spécialistes de sciences politiques et d'autres disciplines ont abordé la notion de politique transnationale a largement évolué au cours des trente dernières années. Joseph Nye et Robert Keohane, qui ont popularisé le terme "transnational relations » au début des années soixante-dix ${ }^{17}$, ont délibérément réagi contre le paradigme "Réaliste " bien connu en relations internationales ${ }^{18}$, selon lequel les organisations internationales «ne sont que les instruments des gouvernements, et n'ont pas d'importance en tant que telles ${ }^{19}$. J. Nye et R. Keohane critiquent l'aspect réducteur de cette approche et son hypothèse faisant des Etats des acteurs unitaires, pour proposer une alternative qu'ils ont appelé le paradigme de la politique mondiale $e^{20}$. Leur ouvrage a lancé un débat sur la théorie des relations internationales, lequel est passé par des phases aussi nombreuses qu'excessivement polarisées. Le Réalisme, qui considère les Etats comme les seuls acteurs importants en politique internationale, reste la cible, avouée ou non, d'une grande partie des recherches sur la mobilisation politique transnationale. Il s'agit là d'un ancrage malheureux dans la mesure où il est difficile pour les réalistes de reconnaître l'importance de la politique transnationale et, pour les anti-réalistes, qui sont les plus nombreux dans l'étude des relations transnationales ${ }^{21}$, d'analyser le rôle des Etats.

2 Ce débat a connu plusieurs phases. Après s'être centrés, dans l'ouvrage dont ils ont dirigé la publication, Transnational Relations and World Politics, sur toutes les formes d'activité transnationale - « les contacts, les coalitions, et les interactions dépassant les frontières étatiques qui ne sont pas contrôlés par les organes de politique étrangère centraux des gouvernements " ${ }^{22}-\mathrm{R}$. Keohane et J. Nye ont limité le concept de transnationalisme aux activités internationales des acteurs non-gouvernementaux en distinguant ces derniers des "acteurs transgouvernementaux", un terme qu'ils emploient maintenant pour se référer à "des sous-unités de gouvernements lorsqu'elles agissent de façon relativement autonome par rapport à des instances supérieures en politique internationale " ${ }^{23}$, et des "organisations internationales ", qu'ils définissent comme "des relations à plusieurs niveaux, des normes et des institutions établies entre gouvernements, prescrivant des comportements dans des situations particulières ». Ce concept affiné de "relations transnationales", bien que plus étroit que la vision originale de ces deux auteurs, n'en couvre pas moins un immense terrain. Il s'est avéré utile en attirant l'attention sur «l'augmentation considérable en nombre et en importance des interactions privées internationales lors des dernières décennies et [sur] le nombre bien plus élevé et plus diversifié d'individus et de groupes s'y engageant ${ }^{24}$. Mais il comporte trois effets fâcheusement restrictifs :

Ces travaux, coïncidant avec la découverte, ou la redécouverte, de l'économie politique internationale, ont poussé les spécialistes américains à privilégier les relations transnationales économiques, et notamment, l'entreprise multinationale, comme l'a précisément fait la majeure partie des auteurs de Transnational Relations and World Politics. Même en 1996, l'ouvrage de R. Keohane en collaboration avec Helen Milner se polarise d'ailleurs encore largement sur les facteurs économiques ${ }^{25}$. En conséquence, les chercheurs en relations transnationales n'ont généralement abordé la politique de la contestation, lorsqu'ils s'y sont aventurés, que sous la forme d'une résistance à la pénétration économique transnationale ${ }^{26}$ et n'ont dans l'ensemble étudié les politiques intérieures des Etats que par le biais de la politique étrangère économique. J. Nye et $R$. 
Keohane, (tout en accordant également une certaine attention à la politique de la contestation ${ }^{27}$ ) n'ont reconnu la contestation transnationale que sous l'intitulé étroit de "diffusion d'idées et d'attitudes », qu'ils ont traitées séparément de leur analyse plus poussée du "pluralisme international», pris comme "l'association de groupes d'intérêts nationaux dans des structures transnationales faisant généralement intervenir des organisations transnationales à des fins de coordination" ${ }^{28}$. Cette séparation entre l'étude de la contestation transnationale et celle des organisations non-gouvernementales transnationales a persisté, ce qui a empêché, jusqu'aux années quatre-vingt-dix, l'intégration des disciplines de la politique transnationale et de celle de la contestation. En troisième lieu, l'accent mis par J. Nye et R. Keohane (sur l'indépendance de l'interaction transnationale) implique, bien qu'ils ne l'aient pas explicitement déclaré, que l'activité transnationale s'effectue aux dépens des Etats nationaux. Cette conclusion, vigoureusement combattue par Huntington dans sa critique de leur ouvrage ${ }^{29}$, laisse non formulées certaines questions sur le rôle des Etats dans la politique transnationale: Quand les Etats stimuleront-ils l'activité transnationale dans leur intérêt et au nom de quels groupes d'intérêts nationaux ? Quand créeront-ils des institutions internationales offrant un forum à des acteurs nongouvernementaux? Quand proposeront-ils en matière d'activité transnationale des modèles reflétant leur propre conception du monde? Et quand promouvront-ils les intérêts d'acteurs non-gouvernementaux contre ceux des autres Etats ${ }^{30}$ ? En résumé trois facteurs se sont combinés pour exclure l'étude de la politique de la contestation du domaine des relations transnationales pendant les années soixante-dix et quatrevingt, à savoir la limitation de l'attention des chercheurs à la seule économie politique et notamment aux entreprises multinationales, la séparation de l'étude de la politique de la contestation d'avec celle des organisations transnationales et l'hypothèse d'un jeu à somme nulle entre Etats et organisations transnationales. Ils peuvent en partie expliquer la stagnation de cette discipline après sa fondation par J. Nye et R. Keohane au début des années soixante-dix, dans la mesure où un grand nombre d'acteurs nongouvernementaux nouveaux à l'époque, mais devenus éminents depuis lors, contestent ouvertement le pouvoir des Etats et des institutions internationales. C'est à la fin de la guerre froide et à la diffusion considérable des organisations non-gouvernementales transnationales dans les années quatre-vingt et quatre-vingt-dix, qu'il faut imputer la reprise et les nouvelles orientations de l'étude de la politique transnationale. Cette évolution se traduisit par deux courants de recherches, l'un émanant de sociologues institutionnalistes comme John Meyer et ses associés à Stanford ${ }^{31}$ et l'autre d'un groupe moins cohérent de politistes davantage unis par ce qu'ils refusaient (entre autres le Réalisme, ou la théorie du choix rationnel) que par ce qu'ils affirmaient. Ces ouvrages offrent une perspective nouvelle de par la provenance étrangère à la sous-discipline des relations internationales d'une grande partie d'entre eux - certains étant produits par d'anciens militants de mouvements pour la paix et d'autres par des spécialistes de la politique et la sociologie de la contestation. Le titre du volume paru sous la direction de Thomas Risse-Kappen en 1995, Bringing Transnational Relations Back In, a révélé la stagnation de cette discipline au cours de la décennie précédente tout en tentant de l'ouvrir à des perspectives allant au-delà du vieux débat entre Réalistes et nonRéalistes. Leur ouvrage et les recherches qu'ils ont inspirées font état en particulier de deux changements notables: 閥 une tentative délibérée de traiter les points d'intersection entre relations transnationales et "structures nationales», et 范 un concept de relations transnationales connoté de façon plus normative. Structures 
nationales et relations transnationales J. Nye et R. Keohane, et surtout ce dernier, ont longtemps souhaité attirer davantage l'attention sur les sources nationales de la politique transnationale (voir notamment l'allocution présidentielle de R. Keohane à l'International Studies Association dans Keohane 1989). T. Risse-Kappen et ses collaborateurs se sont délibérément attaqués à ce problème. "Dans un contexte international équivalent, écrit-il, ce sont les différences de structures nationales qui déterminent la variation de l'influence politique des acteurs transnationaux ${ }^{32}$. Selon eux, pour gagner en influence, les acteurs transnationaux doivent, d'abord, avoir accès au système politique de leur pays cible, et, ensuite, mettre sur pied et/ou participer à des coalitions autour d'une politique publique capables de parvenir à leurs fins. L'approche de T. Risse-Kappen et de ses collaborateurs, contrairement à la «reconnaissance " générique de facteurs nationaux dans les ouvrages antérieurs de relations internationales, a développé des hypothèses sur la façon dont les variations des structures nationales affectent l'influence des acteurs transnationaux. T. RisseKappen soutient par exemple que les sociétés pluralistes seront plus accueillantes pour ces acteurs que les systèmes et les sociétés fermés et hiérarchisés. Comme l'a toutefois montré Matthew Evangelista, le besoin de formation de coalitions dans les systèmes dits ouverts peut constituer un obstacle considérable pour les acteurs transnationaux une fois qu'ils obtiennent une certaine emprise, alors qu'inversement, le système soviétique "fermé », plus difficile d'accès aux partisans du désarmement nucléaire à l'échelon transnational, leur permettait d'exercer une grande influence une fois les contacts établis ${ }^{33}$. L'argument de la "structure nationale » tel que l'ont articulé RisseKappen et ses collaborateurs comportait trois points faibles: 匔 Il était de nature extrêmement générale dans la mesure où il incluait des éléments aussi vastes que la

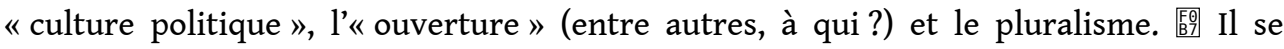
montrait incapable de prévoir la raison du succès de certains acteurs transnationaux et de l'échec de certains autres opérant dans un même contexte ${ }^{34}$. 蔔 Enfin, il ne faisait pas de distinction claire entre différents types d'acteurs transnationaux en regroupant indifféremment en vrac les OING, les mouvements sociaux et les réseaux transnationaux de revendication. Les chercheurs qui ont suivi la lignée des travaux de T. Risse-Kappen et de ses collègues après 1995 ont proposé des éléments de réponse à ces problèmes, grâce à une orientation constructiviste mettant l'accent sur la résonance entre les objectifs transnationaux et les normes nationales. Normes et identités collectives L'évolution de l'étude du militantisme transnational autour de la notion de normes a procédé d'une découverte générale du «constructivisme » par les spécialistes des relations internationales dans les années quatre-vingt-dix ${ }^{35}$. Ces normes furent définies dans divers domaines des relations internationales comme " un modèle de conduite appropriée pour des acteurs ayant une identité donnée " ${ }^{36}$, ce qui vint rallumer la controverse avec le Réalisme et lui donner une nouvelle orientation. Les Réalistes orthodoxes avaient considéré que les intérêts des Etats étaient à la fois fixes et centraux pour les relations internationales. Les néo-Réalistes estimaient que les acteurs transnationaux pouvaient influencer le système international en pesant sur les politiques des Etats les plus forts. Les anti-Réalistes pensaient que s'il pouvait être démontré que les normes ont un rôle autonome en structurant le débat international indépendamment des politiques des Etats forts, la notion d'Etats hégémoniques pouvait être remise en cause. Dans sa forme la plus aboutie, c'est l'argument de ceux qui envisagent que la "gouvernance " transnationale supplantera les États dans les relations internationales. Cette intérêt théorique pour les normes a recoupé l'accent 
mis sur les constructions identitaires du nouvel institutionnalisme en sociologie. John Boli et George Thomas ainsi que leurs collaborateurs, originaires du département de sociologie de Stanford, considèrent la création d'OING, l'évolution vers une plus grande standardisation et la rationalisation des institutions comme des signes de l'émergence d'une culture mondiale dotée d'éléments normatifs fortement universalistes ${ }^{37}$. Les ouvrages de ces chercheurs ont expliqué l'apparition et démontré quantitativement la croissance d'institutions internationales qu'on pouvait considérer comme l'incarnation de nouvelles normes et de nouvelles identités qui reflètent les changements normatifs à l'échelon mondial. L'accent mis sur la notion de normes et d'identité au sein du système international a donné naissance à toute une série de recherches originales : Il a pu être démontré que le consensus normatif transnational a entraîné l'élaboration d'accords internationaux pouvant avoir une action coercitive sur les Etats ${ }^{38}$. Des accords internationaux normatifs ont créé des opportunités politiques pour des acteurs nationaux vivant sous des régimes qui seraient sinon réticents à tolérer la dissidence ${ }^{39}$. Même là où le consensus normatif international n'existait pas, des Etats forts ont pu doter des institutions internationales de l'autorité nécessaire pour faire observer des comportements cohérents avec ces normes, comme c'est le cas des interventions de l'ONU et de l'OTAN en Yougoslavie. 厲 Enfin, les normes ont pu contribuer à la construction de nouvelles identités, qui ont, dans certains cas, pu relier les identités nationales, fournissant une base normative pour les coalitions transnationales ou les réseaux transnationaux fondés sur la défense de principes. A l'image toutefois du paradigme constructiviste, le problème consistant à localiser les normes dans les relations transnationales n'a pas toujours été élucidé. Sont-elles le fait des Etats qui les font passer à d'autres par la persuasion, la force ou l'autorité morale? Sont-elles incarnées par des institutions internationales? Un sceptique n'aurait pas tort de conclure à la lecture des ouvrages sur la question qu'elles naissent comme Vénus, d'une société internationale ou planétaire dans laquelle, sans contraintes ni ressources apparentes, des individus et des groupes de tous les coins du monde s'unissent autour d'un consensus normatif. Il manquait bel et bien un modèle précis des mécanismes de diffusion et de transformation des normes ${ }^{40}$. Les écrits sur les identités transnationales ont buté sur un problème semblable. Les identités peuvent être au moins de deux sortes, à savoir imbriquées dans les processus quotidiens et les réseaux de proximité, ou détachées de ceux-ci et rattachées aux processus politiques et institutionnels ${ }^{41}$. La croissance des organisations transnationales pointe directement vers la création d'identités élitaires « détachées » mais ce mouvement est bien loin de transformer les identités «imbriquées" des citoyens ordinaires au-delà de leurs réseaux interpersonnels. Même en Europe occidentale, où les processus transnationaux institutionnalisés sont les plus denses, il existe peu de preuves du développement d'une identité européenne mis à part le cas des élites qui participent à des conférences internationales, lisent les journaux d'autres pays que le leur et sont membres d'organisations ou de réseaux transnationaux ${ }^{42}$. Un dernier problème est constitué par le fait que la majeure partie des travaux récents sur le militantisme transnational se centre sur des "questions de normes et de principes». Or qu'en est-il de la quantité considérable d'activité transnationale motivée par l'intérêt matériel ? Les entreprises, associations professionnelles et syndicats opérant par delà les frontières ont-ils le même rapport aux normes et à l'identité internationales que les groupes de défense des droits de l'homme, les écologistes et les militants pour la paix? L'occurrence simultanée de la redécouverte de la politique transnationale et du virage 
constructiviste a conduit maints chercheurs en relations internationales à s'intéresser à des réseaux de militants fortement axés sur des questions normatives et à ignorer ceux qui sont mus par des intérêts matériels prédominants, comme les réseaux internationaux de syndicats ${ }^{43}$. Il n'en demeure pas moins que cette nouvelle orientation théorique des relations internationales a eu une conséquence positive inattendue dans la mesure où elle a contribué à jeter un pont entre les relations internationales et une discipline ayant une tradition auparavant distincte, la politique de la contestation, laquelle ne s'était souciée jusqu'à récemment que des mouvements sociaux nationaux ${ }^{44}$. Ce groupe de chercheurs avait déjà absorbé et profité du constructivisme ${ }^{45}$. Il disposait également d'une tradition bien établie d'étude des acteurs s'engageant à la frontière entre politique institutionnelle et mouvement contestataire. Penchons-nous sur les apports des chercheurs de cette tradition à la nouvelle politique transnationale. La politique transnationale de la contestation Ce sont des événements de politique internationale qui ont influencé le développement de cette nouvelle approche. Les événements politiques en ont fourni quatre sources principales: 盛 Des révoltes locales, comme celle de Chiapas, qui articulent leurs revendications de façon mondiale et jouissent du soutien international de groupes nationaux sympathisants et d'OING. 區 Des manifestations internationales de protestation comme la «bataille de Seattle » qui réunissent des coalitions de groupes nationaux et transnationaux contre des cibles extrêmement visibles comme l'Organisation mondiale du commerce et le FMI. 圈 Les succès de certaines coalitions de militants transnationaux contre certains Etats nationaux dans certaines situations. 臨 Enfin, le militantisme au sein et autour d'institutions internationales et de la rédaction internationale de traités. L'avantage de ce nouveau courant de recherche est qu'il attirait un nouveau type de chercheurs. D'anciens militants, qui ont apporté à la discipline leur énergie, des renseignements en temps réel et leur engagement dans l'étude de la contestation, constituent une source importante de données sur la contestation transnationale, comme il le font dans l'étude de mouvements sociaux nationaux. De plus, de perspectives provenant de la politique comparée, de l'anthropologie culturelle et de la sociologie ont enrichi une discipline qui avait été limitée à des spécialistes de relations internationales. Cependant, malgré l'accumulation de connaissances sur la contestation transnationale, il devint vite clair qu'un consensus ne se dégageait pas quant au champ que le terme recouvrait. Par exemple, les révoltes populaires comme celle de Chiapas sont liées à une contestation fondamentalement nationale ayant une articulation transnationale et jouissant du soutien international ${ }^{46}$. Le second type dépend largement d'opportunités et de ressources nationales et internationales particulières et s'avère difficile à maintenir dans la durée, comme l'a prouvé la faillite de la manifestation qui a suivi les événements de Seattle à Washington. Le troisième type, les luttes contre certains Etatsnations qui transgressent les normes internationales, résulte principalement de coalitions élitaires s'appuyant sur l'influence de pays tiers ou d'institutions internationales. Le quatrième type implique des militants transnationaux dans des relations de coopération avec des Etats et des institutions internationales et ne revêt pas forcément un caractère contestataire. Ces nouveaux travaux peuvent se diviser grosso modo en cinq catégories, qui se recoupent parfois quelque peu: Certains ont examiné le développement d'un large spectre d'acteurs non-étatiques qui se sont organisés de façon transnationale ${ }^{47}$. D'autres se sont focalisés sur des familles particulières de mouvements, comme les mouvements pacifistes ${ }^{48}$, de défense des droits 
de l'homme ${ }^{49}$, de protection de l'environnement ${ }^{50}$, ou d'opposants à la construction de barrages ${ }^{51}$, ou encore des mouvements de peuples indigènes ${ }^{52}$. Certains ont centré leurs travaux sur des organisations, soit en étudiant une de celles-ci en particulier ${ }^{53}$, soit en les analysant dans leur ensemble ${ }^{54}$, ou sur des réseaux transnationaux d'organisations ${ }^{55}$. D'autres chercheurs ont étudié les traités internationaux qui ont légitimé et fourni des ressources à des acteurs non-étatiques ${ }^{56}$, ou dans lesquels les militants ont joué un rôle primordial ${ }^{57}$ ou encore contre lesquels ils se sont mobilisés ${ }^{58}$. D'autres enfin se sont penchés sur des mouvements de contestation particuliers binationaux ou régionaux dans le contexte d'accords ou d'institutions internationales ${ }^{59}$. L'étude de la politique transnationale, à l'origine une discipline fortement influencée par les relations économiques transnationales et réduite à un débat quelque peu stérile avec les tenants du Réalisme américain, a commencé à recouper de façon croissante celle de la politique de la contestation. Mais il en va comme dans tout mariage entre chercheurs originaires de traditions différentes : les hypothèses ne sont pas toujours les mêmes et l'adoption du langage et des conventions de l'autre peut susciter des malentendus. L'utilisation du langage de la "mondialisation" dont les connotations économiques, politiques et culturelles se conjuguent de façon changeante a constitué le problème le plus fréquent. La fusion des diverses significations de la mondialisation représente un outil important pour le cadrage des mouvements sociaux, en permettant à leurs organisateurs de s'inscrire dans des mouvements plus larges et d'avoir accès à des ennemis distants en mobilisant des sympathisants. Mais l'adoption de cette terminologie par les chercheurs a été suivie de deux effets néfastes, une indifférence au champ régional d'une grande partie de l'activité transnationale, et une difficulté conceptuelle concernant la distinction entre le cadrage mondial d'une activité et son champ d'action empirique ${ }^{60}$. Quant aux institutions internationales, les militants les ont considérées comme les cibles des mouvements protestataires et non, comme c'est souvent le cas, comme les alliés des acteurs non-étatiques. De surcroît, un grand nombre d'analystes, parvenus à l'étude de la contestation transnationale à partir d'un engagement dans des types de mouvements sociaux particuliers (notamment dans les mouvements pour la paix, l'environnement, les droits des populations indigènes et les mouvements féministes), ont considéré l'univers des acteurs non-étatiques par le biais de "leur" secteur particulier. Ils ont aussi eu tendance à se polariser sur les "bons" mouvements, comme ceux en faveur de la paix et des droits de l'homme, accordant moins d'attention aux secteurs plus dangereux du militantisme transnational, comme par exemple l'extrémisme religieux militant ${ }^{61}$. Enfin, les chercheurs étudiant le militantisme international après s'être cantonnés à l'échelon national ont souvent transféré de façon quelque peu floue la notion connotée idéologiquement de «mouvement social ", à des activités internationales qui seraient davantage assimilables à du lobbying, de la communication et des activités de service et d'enseignement si on les observait dans un cadre national ${ }^{62}$. Les formes du militantisme transnational Nous voici ainsi conduits à un problème à la fois théorique et empirique dans l'étude de la politique transnationale, à savoir la nature et la variété des acteurs sur la scène transnationale. S'agit-il de mouvements sociaux? D'organisations internationales nongouvernementales (OING) ? Ou d'une configuration plus floue comme les « réseaux transnationaux de revendication »? Leur interaction avec les Etats et les institutions internationales pouvant s'en trouver différente, il faut tout d'abord donner une définition élémentaire de ces termes. Les mouvements sociaux transnationaux La nouvelle discipline de la politique transnationale a connu au moment de son apparition 
une tendance croissante à qualifier tout ou la majorité des acteurs transnationaux de " mouvements sociaux " ${ }^{63}$, dont on pouvait mesurer les activités à travers le nombre d'organisations transnationales de mouvements sociaux (OTMS). Certains auteurs se sont penchés sur des organisations transnationales particulières, comme Greenpeace ${ }^{64}$, alors que d'autres ont choisi des échantillons d'OMTS à partir de la catégorie plus large des OING d'après les archives de l'Union des Associations internationales. C'est la possibilité de consulter cette banque de données qui a, de fait, contribué à donner forme à leurs recherches. Smith, Chatfield et Pagnucco définissent les OTMS comme un sous-ensemble des OING opérant dans plus de deux Etats et œuvrant à "changer certains éléments de la structure sociale et/ou la répartition des bénéfices de la société ${ }^{65}$. Il s'agit là d'une définition qui pose problème du fait de l'amplitude de ses paramètres pouvant inclure des groupes aussi divers que la Quatrième Internationale, la World Wildlife Federation, et la Croix Rouge Internationale. Elle ne tient pas non plus compte des types d'activités dans lesquelles ces groupes sont engagés ${ }^{66}$. Comme l'observent Margaret Keck et Kathryn Sikkink, «nous devons comprendre les logiques et les processus très divers des différentes catégories d'acteurs transnationaux pour saisir la façon dont la politique mondiale change " ${ }^{67}$. La classification des acteurs transnationaux en "mouvements sociaux " donne un parfum sulfureux à toute une série d'activités par ailleurs banales mais elle rend difficile la distinction des nombreuses formes que peut revêtir la contestation transnationale. Prenons les syndicats: on s'est de plus en plus penché ces dernières années sur les flux internationaux de capitaux et sur la sous-traitance transnationale et son effet sur les travailleurs ${ }^{68}$. En conséquence, les syndicalistes se sont impliqués dans des activités transnationales bilatérales et multilatérales. Une grande partie de leurs actions concerne étroitement le changement social, mais le fait de les considérer comme des "mouvements sociaux transnationaux» au même titre que Greenpeace, les militants extrémistes musulmans et la Troisième Internationale ne fait guère avancer la réflexion. L'étude de la contestation nationale apporte une solution à ces problèmes de définition en catégorisant les mouvements sociaux non pas selon leur objectif de changement social, un but qu'ils partagent avec un grand nombre de non-mouvements sociaux, mais selon le type d'actions dans lesquelles ils s'engagent habituellement, et en les considérant comme partie intégrante de l'univers plus large de la politique de la contestation, que je définis comme: L'interaction épisodique et collective entre des auteurs de revendications et leur cible a) lorsqu'au moins un gouvernement formule ces revendications, en est la cible ou est partie prenante, et b) dans le cas où leur satisfaction éventuelle doive affecter les intérêts d'au moins un de leurs auteurs ${ }^{69}$. Les mouvements sociaux sont une forme de contestation particulièrement figée dans cet univers et que je définis comme: Des groupes socialement mobilisés engagés dans une interaction soutenue de contestation avec les détenteurs du pouvoir, dans laquelle au moins un des acteurs est soit une cible, soit un participant ${ }^{70}$. Cela nous amène à la définition suivante des mouvements sociaux transnationaux : Des groupes socialement mobilisés ayant des membres dans au moins deux pays, engagés dans une interaction soutenue de contestation avec les détenteurs du pouvoir d'au moins un pays autre que le leur, ou contre une institution internationale ou un acteur économique multinational. Comme toutes les définitions, celle-ci peut pêcher dans un sens ou dans un autre. Mais elle comporte trois avantages. En premier lieu, elle distingue l'action transnationale soutenue des autres formes d'action, comme par exemple un échange politique occasionnel ou la diffusion de la contestation. En second lieu, elle souligne les 
actions de contestation, laquelle ne caractérise pas, loin s'en faut, tous les acteurs nonétatiques. Et, enfin, elle insiste sur l'interaction avec des acteurs en dehors du pays de l'initiateur de contestation, ce qui les sépare des groupes nationaux qui peuvent formuler leurs revendications en termes planétaires mais n'ont pas de lien avec des acteurs au-delà de leurs frontières. Cette définition possède l'avantage stratégique de nous permettre d'examiner comme une question empirique les relations entre les mouvements sociaux et les autres formes d'institutions, principalement constituées par les OING et les réseaux transnationaux de revendication, et de détecter les transitions potentielles entre ces diverses formes.

4 Les organisations internationales non-gouvernementales Qu'il y ait une croissance rapide des organisations non-gouvernementales internationales est un des truismes de la politique transnationale. John Boli et George $\mathrm{M}$. Thomas en énumèrent près de 6000 fondées entre 1875 et $1988^{71}$. Ils ont non seulement observé une accélération du rythme de leur fondation après 1945, mais aussi une diminution de celui de leur dissolution. Il est surprenant de voir combien l'on s'accorde peu sur la définition ou le mode de fonctionnement des OING pour un terme devenu fort courant lors de récents débats ${ }^{72} \mathrm{~J}$. Boli et G. M. Thomas proposent trois descriptions : ils considèrent les OING, d'abord, comme « le champ organisationnel premier dans lequel la culture mondiale prend une forme structurelle " ${ }^{73}$, ensuite comme " des organismes transnationaux exerçant un type spécial d'autorité que nous appelons volontarisme ${ }^{74}$ et enfin comme des groupes dont «la préoccupation première est de faire naître, de codifier, de modifier et de propager des structures et des principes en matière de culture mondiale " ${ }^{75}$. Leur définition opérationnelle est la suivante: "l'ensemble des OING classées comme des organismes authentiquement internationaux par l'Union des Associations internationales ", c'est-à-dire " toutes les organisations non-étatiques non lucratives " ${ }^{76}$. Il s'agit là d'une catégorie extrêmement riche et variée, mais fort peu discriminatoire, ce qui laisse place, pour le moins, à un grand nombre d'incohérences et de lacunes ${ }^{77}$. Je vais risquer une définition qui sera moins vaste tout en distinguant les OING des mouvements sociaux: Les organisations internationales nongouvernementales sont des organisations indépendantes des gouvernements, composées d'une base d'adhérents originaires de plus de deux pays, organisées pour promouvoir les objectifs internationaux de leurs membres et fournir des services aux citoyens d'autres pays par le biais de leurs interactions répétées avec des Etats, des acteurs privés et des institutions internationales. A partir de cette définition, la principale distinction entre les OING et les mouvements sociaux concerne avant tout leur type d'action. Tous deux peuvent vouloir le changement social, mais les mouvements sociaux transnationaux s'engagent dans une interaction soutenue de contestation avec les Etats, les acteurs multinationaux ou les institutions internationales, tandis que les OING ont des rapports plus institutionnalisés avec ces mêmes types d'acteurs et fournissent des services à des citoyens d'autres pays. L'établissement d'une distinction analytique claire entre ces deux catégories d'action facilite l'examen des relations qu'elles ont entre elles, de façon à pouvoir déterminer l'existence éventuelle de transitions d'un type à l'autre, ainsi que la comparaison de leur relation avec les mouvements sociaux de base. Ce dernier point est particulièrement important. Les OING sont largement composées d'élites, de personnes dévouées qui ont les moyens de voyager de par le monde, parlent des langues étrangères et possèdent les qualifications techniques, professionnelles et intellectuelles leur permettant de servir et de représenter les intérêts de ceux qu'ils soutiennent 
auprès des institutions internationales et des Etats les plus puissants. Bien que les mouvements sociaux aient également besoin de dirigeants, et se soient davantage professionnalisés pendant les dernières décennies ${ }^{78}$, ils se basent, au moins selon notre définition, sur des "groupes socialement mobilisés engagés dans une interaction soutenue de contestation avec les détenteurs du pouvoir ». Ce ne sont pas les mêmes types d'acteurs que les dirigeants d'OING et la question des relations entre ces deux catégories est d'ordre empirique. Les réseaux transnationaux de militants Une des raisons pour lesquelles les OING font l'objet d'une abondante littérature est que leur naissance et leur mort, leurs structures organisationnelles et leurs liens institutionnels sont officiellement enregistrés et se trouvent recensés en détail par des organisations dont l'UAI et les Nations Unies. Elles constituent une mesure utile des changements des organisations internationales ${ }^{79}$, malgré l'aspect brut des données les évaluant ${ }^{80}$. Or les spécialistes ont récemment pris conscience du fait qu'excepté leurs activités de service pour lesquelles elles restent habituellement indépendantes, les OING fonctionnent souvent à l'aide d'alliances temporaires ou de longue durée avec d'autres acteurs - tant étatiques que non-étatiques, transnationaux que nationaux - pour promouvoir leurs objectifs. Cette constatation a enrichi l'étude de la politique transnationale d'une catégorie nouvelle et dynamique, les réseaux transnationaux de militants. Comme le définissent $M$. Keck et $K$. Sikkink : «Un réseau transnational de militants inclut des acteurs travaillant sur une question à l'échelle internationale, liés par des valeurs partagées, un discours commun et des échanges ${ }^{81} \cdot{ }^{82}$ denses d'information et de services $1{ }^{83}$ Les réseaux transnationaux de militants ne sont pas des alternatives aux mouvements sociaux ni aux OING mais peuvent au contraire les contenir, de la façon lâche dont les réseaux contiennent quoi que ce soit, comme ils peuvent également inclure des fonctionnaires gouvernementaux ou internationaux. Ces réseaux constituent les structures informelles et changeantes par lesquelles les ONG, les militants des mouvements sociaux, les responsables gouvernementaux et le personnel des institutions internationales peuvent entrer en contact et aider des acteurs nationaux pauvres en ressources à peser politiquement au sein de leur propre société. Dans le modèle de M. Keck et $\mathrm{K}$. Sikkink, les ONG riches en ressources tentent d'activer des réseaux transnationaux pour faire pression sur le pays cible, en travaillant avec leurs propres Etats, les institutions internationales, ou les deux. Le concept de réseaux transnationaux de militants développé par M. Keck et $\mathrm{K}$. Sikkink représente une avancée à la fois conceptuelle et empirique. Les militants dont elles traitent sont souvent des représentants des ONG mais pas seulement; les relations qu'ils entretiennent avec les Etats et les institutions internationales ont des facettes multiples et changeantes : parfois ils les attaquent mais souvent ils coopèrent avec eux. C'est en cela que leur livre marque un progrès par rapport à ceux qui chantent les louanges d'une « société civile globale ». Néanmoins, leur ouvrage soulève un certain nombre de problèmes: 盛 Leur vision des relations entre les réseaux transnationaux de militants et les systèmes étatiques existants n'apparaît pas clairement. Le fonctionnement de ces réseaux dépend-il incidemment (ou fondamentalement) de la puissance des Etats dont ils sont originaires? La majorité de leurs groupes d'adhérents provenant des pays riches du Nord, la puissance de ces pays joue-t-elle pour permettre aux militants de ces réseaux de persuader d'autres pays d'accéder aux revendications de leurs alliés pauvres en ressources? Quelle est la relation entre les militants de ces réseaux et les mouvements sociaux nationaux - souvent victimes de la répression étatique ? Lorsque leurs efforts portent leurs fruits à court terme, ces réseaux sont susceptibles d'avoir un 
effet positif. Mais quels changements structurels et comportementaux ont-ils à leur actif lorsqu'ils se dispersent une fois la campagne terminée ? Leurs alliés locaux ont-ils acquis les moyens de constituer une opposition plus forte au pouvoir établi ou se retrouvent-ils isolés et à la merci de gouvernements vengeurs ${ }^{84}$ et diffusés ensuite au niveau national. On peut prendre en défaut cette vision d'une société civile mondiale émergente produisant des actions collectives transnationales tant elle dérive par trop étroitement de la "mondialisation » et tant elle se trouve insuffisamment spécifiée. En ce qui concerne la première objection, j'ai déjà soutenu que l'existence de nombreuses activités d'organisations transnationales a précédé la phase actuelle d'interdépendance économique mondiale. Mais il existe un problème plus sérieux dans la mesure où ce modèle ne propose pas de mécanisme permettant de surmonter les obstacles que rencontrent les citoyens ordinaires en matière d'action collective transnationale. Ces obstacles sont de trois sortes : la faiblesse ou l'absence de réseaux sociaux en dehors des quartiers, des villes, des grands centres urbains, des groupes sociaux et des allégeances politiques; la faiblesse ou l'absence d'identité collective transnationale ; et l'absence de mécanismes permettant de surmonter ou de contrer les opportunités politiques des instances politiques nationales ${ }^{85}$. Imig et Tarrow ont par exemple relevé qu'en Europe occidentale, plus de $80 \%$ des actions protestataires étudiées étaient dirigées contre des acteurs ou des institutions infranationales même lorsque l'Union européenne constituait la source ou la cible de la contestation ${ }^{86}$. Tout en gardant dans bien des domaines un certain parallélisme avec la "société civile mondiale", le courant institutionnaliste met en avant une intensification de la contestation transnationale par le biais des ressources, des incitations et des opportunités de l'interaction internationale. Comme le soutient Thomas Risse, «plus le degré d'institutionnalisation internationale dans un domaine donné est élevé, plus l'influence politique des acteurs transnationaux est importante ${ }^{87}$. Il approfondit ce thème dans un récent article : «Les acteurs transnationaux prendront d'autant plus d'importance qu'ils agiront dans un environnement international fortement structuré par des institutions et des régimes de gouvernance internationales. Par exemple, les organisations internationales constituent des enceintes permettant des interactions régulières entre acteurs des réseaux transnationaux de revendication et acteurs étatiques. Dans certains cas, ils encouragent (et même financent) activement les OING et d'autres coalitions transnationales" ${ }^{88}$. L'institutionnalisation peut revêtir différentes formes, des conventions internationales à des institutions formelles en passant par des structures lâches ${ }^{89}$. Elle varie en intensité selon le secteur d'activité. Elle est dynamique et traverse différentes phases, dont certaines sont propices à l'influence d'acteurs transnationaux - ce que Martha Finnemore et Kathryn Sikkink appellent par exemple une phase d' " émergence des normes " ${ }^{90}$ - et dont certaines ont des objectifs restreints, empêchant les acteurs non-étatiques de peser de tout leur poids. De fait, l'émergence et la longévité des acteurs non-étatiques transnationaux varieront de secteur en secteur dans la mesure où leur vigueur dépend d'institutions internationales, et pourront même se trouver en perte de vitesse au fur et à mesure que ces institutions viendront à maturité ou perdront de l'importance. L'approche institutionnelle de la contestation transnationale identifie plusieurs mécanismes qui permettent aux militants nationaux de se rencontrer, de gagner en légitimité, de construire des identités collectives et de retourner dans leurs pays forts de nouvelles alliances, de programmes communs, et de moyens d'action collective inédits. On peut identifier au moins quatre mécanismes de ce type : le courtage, la certification, la modélisation et l'appropriation institutionnelle. 
Une définition élémentaire de ces termes est ici nécessaire : 圆 J'entends par courtage le fait de mettre en relation des acteurs qui seraient sinon isolés d'une façon à créer au moins une identité politique temporaire qui n'existait auparavant. 㲘 Je définis la certification comme la reconnaissance des identités et de l'activité publique légitime d'acteurs nouveaux ou nouveaux à un type particulier d'activité. 區 J'appelle modélisation l'adoption dans un contexte particulier de normes, de formes d'action ou d'organisation collective qui avaient été employées à une autre occasion. 蔔 Je conçois l'appropriation institutionnelle comme l'utilisation des ressources ou de la réputation d'une institution pour servir les objectifs des groupes qui lui sont affiliés. Prise isolément, aucune organisation internationale ne pourra fournir les mécanismes capables de faciliter tous ces processus, et, certes, la majorité d'entre elles est fort loin de s'en approcher. Mais la liste fournie ci-dessus peut sans doute aider les chercheurs à spécifier les façons dont les acteurs non-étatiques dotés de peu de ressources et d'opportunités au sein de leur propre société sont à même de développer des liens transnationaux pouvant faire l'objet d'un "effet boomerang" et soutenir leurs revendications. Cette typologie peut également servir de pistes à la recherche empirique dans des zones d'institutionnalisation internationale comme l'Union européenne. Des européens contestataires ${ }^{11}$ Les chercheurs «transnationalistes » qui examinent les processus que nous venons de décrire ont beaucoup à apporter à ceux qui étudient l'intégration européenne. Il est cependant frappant, qu'à l'exception de quelques clins d'œil à la « mondialisation », les spécialistes de l'intégration européenne ignorent largement le débat mené à l'heure actuelle dans le domaine de la politique transnationale sur l'augmentation des mouvements protestataires qui traversent les frontières. Cela peut s'expliquer par le sentiment que l'Europe est un continent différent des autres, en raison des pouvoirs de ses institutions internationales et son niveau de développement économique et social. Mais ce sentiment légitime a rendu les spécialistes de l'intégration européenne si fascinés par leur propre continent qu'ils délaissent la comparaison de l'Union européenne avec des institutions internationales, fort différentes soit, qui émergent ailleurs dans le monde. Considérons le "panel d'inspection » de la Banque mondiale avec Jonathan Fox. La Banque a créé en 1993 un panel d'organisations spécialisées dans l'environnement et les droits de l'homme pour lui prodiguer des conseils sur la transparence politique de ses projets. Est-ce si différent que les consultations habituelles entre les fonctionnaires de la Commission européenne et les acteurs non-gouvernementaux qui gravitent autour de Bruxelles? Nous n'affirmons pas que les processus transnationaux que nous venons d'évoquer dans cet article peuvent s'appliquer tels quels à l'Europe occidentale ni que l'ALENA, les NationsUnies, l'Organisation mondiale du commerce ou la Banque mondiale soient semblables aux institutions européennes. Mais ceux qui s'intéressent à l'émergence d'un champ politique européen - comme c'est le cas des auteurs de ce numéro spécial - feraient bien de réfléchir sur le développement de mouvements sociaux transnationaux, d'ONG et de réseaux de militants dans l'Union européenne à la lumière de leur analogie avec les formes de contestation transnationale que l'on trouve dans d'autres régions du monde. Certaines de ces analogies sont évidentes :

范 Comme les autres institutions internationales, l'UE a été créée par des Etats pour la poursuite d'intérêts communs. 䔽 Comme eux, les Etats européens ont établi un Secrétariat permanent - la Commission européenne - pour gérer leurs affaires communes. 臨 En exerçant l'autorité qui lui a été déléguée pour régler les désaccords entre Etats membres et réguler leur comportement, la Commission a gagné en 
autonomie et a eu intérêt à établir des relations étroites avec des acteurs non-étatiques. 蓲 Ces relations contribuent à renforcer l'autonomie et la légitimité de la Commission dans différents domaines de politique publique tout en encourageant des acteurs nonétatiques à graviter autour de la «barrière de corail » bruxelloise. 圈 Enfin, ces relations dépassent les frontières nationales et entraînent la formation de coalitions transnationales. Par ailleurs, les groupes dont la légitimité et les intérêts ne sont pas reconnus par la Commission européenne manifestent de plus en plus dans les Etats membres contre ses décisions. Jusqu'à présent, peu d'entre eux sont devenus de véritables mouvements sociaux transnationaux, mais des événements comme la collaboration des ouvriers français et belges de Renault pendant la grève à Vilvoorde en 1997, la marche européenne des chômeurs, et les manifestations multinationales d'agriculteurs sont de plus en plus fréquents ${ }^{92}$. Les institutions européennes sont des sources de courtage, de certification, de modélisation et d'appropriation institutionnelle pour les acteurs non-étatiques qui ont pris conscience de leur pouvoir d'intervention et de réglementation dans leurs sociétés.

6 Il est important de mettre en perspective ces tendances. Les "transnationalistes" oublient souvent que la grande majorité des actions protestataires a lieu à l'intérieur des frontières des Etats-nations, et, même lorsque les adversaires se trouvent à l'extérieur des frontières nationales, les actes de résistance contre ces derniers sont le fait d'acteurs sociaux nationaux opérant dans un cadre national. Une étude récente a par exemple montré que la majorité des actions protestataires contre les politiques de l'Union européenne sont dirigées contre les gouvernements nationaux qui les mettent en œuvre. De la même manière, les acteurs politiques agissant au niveau national ne deviennent pas forcément transnationaux quand ils donnent un cadrage mondial à leurs revendications ou reçoivent de l'aide d'OING ou de réseaux de revendication. Cela dépend des secteurs concernés et nécessite un travail de cadrage et d'organisation difficile et contesté ainsi que le savoir et le pouvoir de s'appuyer sur des institutions internationales pour y parvenir. C'est la raison pour laquelle je pense qu'un modèle institutionnel a davantage de poids analytique que le concept, plus spectaculaire, de "société civile mondiale». La "mondialisation» ne peut pas ex nihilo inciter des acteurs non-étatiques faibles et dispersés à se rencontrer et à développer les identités collectives communes et les réseaux nécessaires pour influencer les acteurs les plus puissants sur la scène mondiale. Par contre, les institutions internationales servent de « récif de corail» aux acteurs non-étatiques en contribuant à créer des liens horizontaux au-delà des frontières entre militants ayant des revendications semblables ou complémentaires. D'où le paradoxe selon lequel les institutions internationales, quoiqu'établies par les Etats et généralement les plus puissants d'entre eux, constitue le berceau de la contestation transnationale. Je n'affirme pas que les pays créent des institutions internationales pour encourager la contestation et les Etats sont davantage à même de déléguer leur souveraineté que de la fusionner ${ }^{93}$. Mais les institutions internationales, qui gagnent en autonomie lorsqu'elles jouent un rôle de médiateur face aux intérêts d'Etats en situation de concurrence, peuvent offrir des opportunités politiques à des acteurs sociaux nationaux faibles, en encourageant de leur part l'établissement de liens avec des homologues et en leur fournissant des ressources utilisables dans les conflits infranationaux ou transnationaux. Ces considérations sont tout aussi spéculatives que la citation de Tilly placée en exergue de cet article. Mais elles indiquent des questions de recherche prometteuses, dont certaines ont déjà été posées en Europe et ailleurs: 睌 Quels sont les acteurs nationaux les plus susceptibles de 
former avec leurs homologues par delà les frontières (et autour de questions de quel ordre ?) des réseaux ayant une longue durée de vie et non élitaires? 期 Quels sont les effets à long terme des liens entre les OING et les réseaux transnationaux de militants et leurs alliés nationaux pauvres en ressources? Se substituent-ils au militantisme national? Détachent-ils les militants ambitieux de leurs activités d'organisation à l'échelon national pour les rendre cosmopolites? Ou viennent-ils renforcer le pouvoir des mouvements nationaux? 医 Quel est le rôle des Etats (et particulièrement des pays hégémoniques) comme source d'aide ou comme obstacle aux alliances transnationales? Enfin, peut-on systématiser les relations dynamiques entre les organisations transnationales et les institutions internationales? Boli et Thomas ont montré le parallélisme sur le long terme des taux de croissance de ces deux ensembles de collectivités ${ }^{94}$. Mais peut-on démontrer qu'il s'agit d'une croissance interactive à long terme ou de deux résultats indépendants d'un processus de rationalisation internationale? Enfin, pour conclure, une idée provocatrice: si les processus d' « internationalisation » décrits ci-dessus sont avérés, l'avènement d'une société civile mondiale ne sera pas dû à la tendance des groupes nationaux à se mobiliser au-delà des frontières et à remplacer les gouvernements nationaux par un système de gouvernance basé à Bruxelles. Il proviendra plutôt du reflux de leurs activités vers la contestation, les institutions et les identités nationales. Et si tel est le cas, il faudra remettre en cause la distinction entre relations internationales et politique intérieure !

7 Sources Anner Mark, «Transnational Campaigns to Defend Labor Rights in Central America's Export Processing Plants », article non publié, School of Industrial and Labor Relations, Cornell University, 1998. Arrighi Giovanni et Silver Beverly, «Labor Movements and Capital Migration » in Charles Bergquist (dir.), Labor in the Capitalist World-Economy, Beverly Hills et Londres, Sage, 1984, pp.183-216. Ayres Jeffrey M., Defining Conventional Wisdom : Political Movements and Popular Contention against North American Free Trade, Toronto, University of Toronto Press, 1998. Blyton Paul, Martinez Lucio Miguel, McGurk John et Turnbull Peter, « Globalisation and Trade Union Strategy: Industrial Restructuring and Human Resource Management in the International Civil Aviation Industry", article non publié, Cardiff University Department of Industrial Relations, 2000. Boli John et Thomas John (dirs.), Constructing World Culture: International Nongovernmental Organizations since 1875, Stanford, Stanford University Press, 1999. Brysk Alison, From Tribal Village to Global Village : Indian Rights and International Relations in Latin America, Irvine, University of California, manuscrit non publié, 1998. Della Porta Donatella, Kriesi Hanspeter et Rucht Dieter (dirs.), Social Movements in a Globalizing World, Londres, MacMillan, 1999. Evangelista Matthews, "Transnational Relations, Domestic Structure, and Security Policy in the USSR and Russia », in Thomas Risse-Kappen (dir.), Bringing Transnational Relations Back In, Ithaca et Londres, Cornell University Press, 1995, chap.5. Evangelista Matthews, Unarmed Forces: Transnational Relations and the Demise of the Soviet Threat, Ithaca et Londres, Cornell University Press, 1999. Finnemore Martha, National Interests in International Societies, Ithaca et Londres, Cornell University Press, 1996. Finnemore Martha et Sikkink Kathryn, «International Norm Dynamics and Political Change ", International Organization n 52, 1998, pp.887-917. Fox Jonathan, « Assessing Binational Civil Society Coalitions », Chicago/Latino Research Center Working Paper $\mathrm{n}^{\circ}$ 26, University of California at Santa Cruz, 2000. Huntington Samuel P., "Transnational Organizations in World Politics», World Politics $n^{\circ} 25,1973$, pp. 333-368. Imig Doug et Tarrow Sidney, «Europeanization of Movements? Contentious 
Politics and the European Union, October 1983-March 1995 », in Donatella della Porta, Hanspeter Kriesi et Dieter Rucht (dirs.), Transnational Social Movements, Londres, MacMillan, 1999. Imig Doug et Tarrow Sidney, «Processing Contention in a Europeanising Polity, West European Politics », en cours d'impression, 2000. Imig Doug et Tarrow Sidney, "Mapping the europeanization of contention: Evidence from a quantitative data analysis ", chap.2, in Doug Imig et Sydney Tarrow (dirs.), Contentious Europeans: Protests and Politics in an Integrating Europe, Boulder, Rowman and Littlefield, à paraître. Imig Doug et Sidney Tarrow (dirs.), Contentious Europeans : Protest and Politics in an Integrating Europe, Boulder, Rowman and Littlefield, à paraître. Jacobson Harold K., Networks of Interdependence: International Organizations and the Global Political System, New York, Knopf, 1979. Katzenstein Peter J., "Introduction : Alternative Perspectives on National Security » in Katzenstein (dir.), The Culture of National Security: Norms and Identity in World Politics, New York, Columbia University Press, 1996. Keck Margaret et Sikkink Kathryn, Activists Beyond Borders. Advocacy Networks in International Politics, Ithaca, Cornell University Press, 1998. Keck Margaret et Sikkink Kathryn, "Social Movements and Transnational Advocacy Networks ", in David S. Meyer et Sidney Tarrow (dirs.), Towards a Movement Society? Contentious Politics for a New Century, Boulder, Rowman and Littlefield, 1998. Keohane Robert O., International Institutions and State Power: Essays in International Relations Theory, Boulder, Westview, 1989. Keohane Robert O. et Milner Helen V. (dirs.), Internationalization and Domestic Politics, New York et Cambridge, Cambridge University Press, 1996. Keohane Robert O. et Nye Joseph S. Jr., « Transgovernmental Relations and International Organizations », World Politics $\mathrm{n}^{\circ}$ 27, 1974, pp.39-62. Keohane Robert O. et Nye Joseph S. Jr. (dirs.), Transnational Relations and World Politics, Cambridge, Massachusetts, Harvard University Press, 1971. Khagram Sanjeev, «Transnational Struggles for Power and Water: The Political Economy of Big Dam Building and Development in the Third World ", thèse de doctorat non publiée, Stanford University, département de science politique, 1999. Khagram Sanjeev, Riker James et Sikkink Kathryn (dirs.), Reconstructing World Politics: Transnational Social Movements and Norms, à paraître. Klotz Audie, Norms in International Relation: The Struggle Against Apartheid, Ithaca et Londres, Cornell University Press, 1995. Krasner Stephen, «Power Politics, Institutions and Transnational Relations » chap. 8, in Thomas Risse-Kappen (dir.), Bringing the State Back In: Non-State Actors, Domestic Structures and International Institutions, New York et Cambridge, Cambridge University Press, 1995. Martin, Andrew et George Ross (dirs.) The Brave New World of European Labor: European Trade Unions at the Millennium. New York et Oxford, Berghahn, 2000. McAdam Doug, «On the International Origins of Domestic Political Opportunities ", in Anne Costain et Andrew S. McFarland (dirs.), Social Movements and Political Institutions in the United States, Boulder, Rowman and Littlefield, 1998, chap.15. McAdam Doug, Tarrow Sidney et Tilly Charles, Dynamics of Contention, New York et Cambridge, Cambridge University Press, à paraître. Melucci Alberto, "Getting Involved: Identity and Mobilization in Social Movements ", in Bert Klandermans, Hanspeter Kriesi et Sydney Tarrow (dirs.), From Structure to Action : Comparing Social Movements Across Cultures, International Social Movement Research I, Greenwich, Connecticut, JAI, 1988, pp.329-348. Melucci Alberto, Challenging Codes: Collective Action in the Information Age, Cambridge et New York, Cambridge University Press, 1996. Meyer David et Tarrow Sidney (dirs.), Towards a Movement Society? Contentious Politics for a New Century, Boulder, Rowman and 
Littlefield, 1998. Moravcsik, Andrew, The Choice for Europe : Social Purpose and State Power from Messina to Maastricht, Ithaca, NY, Cornell University Press, 1998. Nye Joseph S. Jr. et Keohane Robert O., "Transnational Relations and World Politics » : "Introduction» et "Conclusion» in Robert O. Keohane et Joseph Nye Jr. (dirs.), Transnational Relations and World Politics, Cambridge, Massachusetts, Harvard University Press, 1971, pp. ix-xxix et 371-398. Price Richard, The Chemical Weapons Taboo, Ithaca et Londres, Cornell University Press, 1997. Risse Thomas, «Transnational Actors, Networks and Global Governance ", in Walter Carlsnaes, Thomas Risse, et Beth Simmons (dirs.), Handbook of International Relations, Londres, Sage, 2000 (en préparation). Risse Thomas, Ropp Stephen C., et Sikkink Kathryn (dirs.), The Power of Human Rights : International Norms and Domestic Change, New York et Cambridge, Cambridge University Press, 1999. Risse-Kappen Thomas (sous la dir.), Bringing Transnational Relations Back In, Ithaca et Londres, Cornell University Press, 1995. Rochon Tom, Mobilizing for Peace : The Antinuclear Movements in Western Europe, Princeton, Princeton University Press, 1988. Rosenau James, Turbulence in World Politics: A Theory of Change and Continuity, Princeton, Princeton University Press, 1990. Rosenau James, "Towards an Ontology for Global Governance», in Martin Hewson et Timothy J. Sinclair (dirs.), Approaches to Global Governance Theory, Albany, State of New York Press, 1999, chap.13. Rucht Dieter, "The Transnationalization of Social Movements: Trends, Causes, Problems » in Donatella della Porta, Hanspeter Kriesi et Dieter Rucht (dirs.), Social Movements in a Globalizing World, Londres, McMillan, 1999, pp.206-222. Rudolf, Hoeber Susanne et Pescatori James (dirs.), Transnational Religion and Fading States, Boulder, Westview, 1997. Sikkink Kathryn et Smith Jackie, «Infrastructure for Change » in Sanjeev Khagram, James Riker et Kathryn Sikkink (dirs.), Reconstructing World Politics: Transnational Social Movements and Norms, chap. 2, à paraitre. Smith Jackie, "Transnational Organizations " in Encyclopaedia of Violence, Peace and Conflict ", $n^{\circ} 3$, 1999, pp.591-602. Smith Jackie, Chatfield Charles et Pagnucco Ron (dirs.), Transnational Social Movements in Global Politics, Syracuse, Syracuse Univerity Press, 1997. Snow David, Rochford Burke E., Worden Steven et Benford Robert, «Frame Alignment Processes, Micromobilization, and Movement Participation ", American Sociological Review n 51, 1986, pp.464-481. Spruyt Hendrik, The Sovereign State and its Competitors, Princeton, Princeton University Press, 1994. Tarrow Sidney, Power in Movement: Social Movements and Contentious Politics, New York et Cambridge, Cambridge University Press, 2ème édition révisée, 1998. Thomas Daniel, The Helsinki Effect, Princeton University Press, Princeton, à paraître. Tilly Charles, "The Time of States », Center for the Study of Social Change Working Paper No 172, New York School for Social Research, 1993. Tilly Charles, Popular Contention in Great Britain, 1758-1834, Cambridge, Massachusetts, Harvard University Press, 1995. Walton John, «Debt, Protest and the State in Latin America » in Susan Eckstein (dir.), Power and Popular Protest : Latin American Social Movements, Berkeley et Los Angeles, University of California Press, 1989. Yashar Deborah J., "Citizenship Claims in Latin America: Parsing out the Role of Globalization", intervention lors de la conférence sur les revendications de citoyenneté, Harvard University Center for International Affairs, octobre 1998. Young Oren (dir.), Global Governance : Drawing Insights from the Environmental Experience, Cambridge, Massachusetts, MIT Press, 1997. 


\section{NOTES}

1. Charles Tilly "The Time of States ", Center for the Study of Social Change Working Paper No 172, New York School for Social Research, 1993, p.3.

2. Oren Young (dir.), Global Governance : Drawing Insights from the Environmental Experience, Cambridge, Massachusetts, MIT Press, 1997.

3. Paul Wapner, Environmental Activism and World Civic Politics, Albany, NY, SUNY Press, 1996.

4. Margaret Keck et Kathryn Sikkink, Activists Beyond Borders. Advocacy Networks in International Politics, Ithaca, Cornell University Press, 1998.

5. Thomas Risse, «Transnational Actors, Networks and Global Governance », in Walter Carlsnaes, Thomas Risse, et Beth Simmons (dirs.), Handbook of International Relations, Londres, Sage, 2000 (en préparation).

6. Stephen Krasner, « Power Politics, Institutions and Transnational Relations » chap. 8, in Thomas Risse-Kappen (dir.), Bringing the State Back In : Non-State Actors, Domestic Structures and International Institutions, New York et Cambridge, Cambridge University Press, 1995. Hendrik Spruyt, The Sovereign State and its Competitors, Princeton, Princeton University Press, 1994. Voir également Thomas Risse, 2000, op.cit. 7. M. Keck et K. Sikkink, Activists Beyond Borders, op.cit.

8. Sidney Tarrow, Power in Movement : Social Movements and Contentious Politics, New York et Cambridge, Cambridge University Press, 2ème édition révisée, 1998. Charles Tilly, Popular Contention in Great Britain, 1758-1834, Cambridge, Massachusetts, Harvard University Press, 1995.

9. James Rosenau, Turbulence in World Politics : A Theory of Change and Continuity, Princeton, Princeton University Press, 1990.

10. Harold K. Jacobson, Networks of Interdependence : International Organizations and the Global Political System, New York, Knopf, 1979. M. Keck et K. Sikkink, Activists Beyond Borders, op.cit., 1998.

11. Samuel P. Huntington, «Transnational Organizations in World Politics », World Politics n² 25, 1973, pp. 333-368.

12. Andrew Moravcsik, The Choice for Europe : Social Purpose and State Power from Messina to Maastricht, Ithaca, NY, Cornell University Press, 1998.

13. Robert O. Keohane et Joseph S. Nye Jr., « Transgovernmental Relations and International Organizations », World Politics n²7, 1974, pp. 39-62.

14. R. O. Keohane, International Institutions and State Power : Essays in International Relations Theory, Boulder, Westview, 1989.

15. S. P. Huntington, op.cit.

16. Une tentative similaire à la mienne se trouve dans T. Risse, « Transnational Actors, Networks and Global Governance ", op. cit.

17. Mais sans l'inventer toutefois. Ils sont redevables en cela à Raymond Aron, Philip Jessop, Karl Kaiser, Horst Menderhause, James Rosenau et Stanley Hoffmann comme ils le reconnaissent dans la préface de Transnational Relations and World Politics paru en 1971 (p. vii), puis, en partie, dans leurs travaux ultérieurs, à leur débat avec Samuel Huntington qui adopta une conception différente, et plus large, des relations transnationales. Cf. R. O. Keohane et J. S. Nye Jr. (dirs.), Transnational Relations and World Politics, Cambridge, Massachusetts, Harvard University Press, 1971, pp. 372-379. 
18. R. O. Keohane et J. S. Nye Jr. (dirs.), Transnational Relations and World Politics, op. cit.

19. R. O. Keohane et J. S. Nye Jr., « Transgovernmental Relations and International Organizations ", op.cit., p. 39.

20. R. O. Keohane et J. S. Nye Jr. (dirs.), Transnational Relations and World Politics, op.cit., pp. 379-395.

21. T. Risse, "Transnational Actors, Networks and Global Governance », op.cit., p. 2

22. R. O. Keohane et J. S. Nye Jr. (dirs.), Transnational Relations and World Politics, op.cit., p. xi

23. R. O. Keohane et J. S. Nye Jr. (dirs.), « Transgovernmental Relations and International Organizations », op.cit., p. 41.

24. Huntington, op.cit., p. 335.

25. R. O. Keohane et Helen V. Milner (dirs.), Internationalization and Domestic Politics, New York et Cambridge, Cambridge University Press, 1996.

26. Giovanni Arrighi et Beverly Silver, « Labor Movements and Capital Migration » in Charles Bergquist (dir.), Labor in the Capitalist World-Economy, Beverly Hills et Londres, Sage, 1984, pp. 183-216. John Walton, « Debt, Protest and the State in Latin America » in Susan Eckstein (dir.), Power and Popular Protest : Latin American Social Movements, Berkeley et Los Angeles, University of California Press, 1989.

27. R. O. Keohane et J. S. Nye Jr. (dirs.), Transnational Relations and World Politics, op.cit., p. xvii.

28. R. O. Keohane et J. S. Nye Jr. (dirs.), ibid., p. xviii.

29. Hungtinton, op.cit, p. 342 et suivantes.

30. Ils comblent cette lacune de façon quelque peu abstraite lorsqu'ils décrivent dans leur article de 1974 « la possibilité d'une intervention gouvernementale dans des systèmes à prédominance non-gouvernementale ». Cf. R. O. Keohane et J. S. Nye Jr., «Transgovernmental Relations and International Organizations », op. cit., p. 55.

31. Voir la bibliographie complète de John Boli et George Thomas (dirs.), Constructing World Culture : International Nongovernmental Organizations since 1875, Stanford, Stanford University Press, 1999.

32. T. Risse-Kappen (dir.), Bringing Transnational Relations Back In, Ithaca NY, Cornell University Press, 1995, p. 25.

33. Matthew Evangelista, «Transnational Relations, Domestic Structure, and Security Policy in the USSR and Russia », in T. Risse-Kappen (dir.), Bringing Transnational Relations Back In, op. cit., chap. 5, et Matthew Evangelista, Unarmed Forces : Transnational Relations and the Demise of the Soviet Threat, Ithaca et Londres, Cornell University Press, 1999.

34. M. Keck et K. Sikkink, Activists Beyond Borders, op.cit., p. 202.

35. T. Risse, "Transnational Actors, Networks and Global Governance », op.cit., p. 2. Pour un compte rendu et des hypothèses stimulantes et des références bibliographiques qu'elles indiquent, cf. Martha Finnemore et K. Sikkink, « International Norms Dynamics and Political Change ", International Organization n 52, 1998, pp. 887-917. Cf. également Martha Finnemore, National Interests in International Societies, Ithaca et Londres, Cornell University Press 1996 ; Peter J. Katzenstein, « Introduction : Alternative Perspectives on National Security » in P. J. Katzenstein (dir.), The Culture of National Security : Norms and Identity in World Politics, New York, Columbia University Press, 1996 ; Audie Klotz, Norms in International Relations : The Struggle Against Apartheid, Ithaca et Londres, Cornell University Press, 1995 ; Richard Price, The 
Chemical Weapons Taboo, Ithaca et Londres, Cornell University Press, 1997 ; et Daniel Thomas, The Helsinki Effect, Princeton, Princeton University Press, à paraître.

36. P. J. Katzenstein, op.cit, p. 5.

37. Boli John et Thomas John (dirs.), op.cit.

38. A. Klotz, op.cit., et R. Price, op.cit..

39. D. Thomas, op.cit. ; T. Risse Thomas, Stephen C., Ropp et K. Sikkink (dirs.), The Power of Human Rights : International Norms and Domestic Change, New York et Cambridge, Cambridge University Press, 1999.

40. Pour une exception, voir Risse, Ropp et Sikkink, op.cit.

41. Doug McAdam, S. Tarrow et Charles Tilly, Dynamics of Contention, New York et Cambridge, Cambridge University Press, à paraître, chap. 5.

42. Doug Imig et S. Tarrow, « Processing Contention in a Europeanising Polity, West European Politics, en cours d'impression, 2000.

43. Paul Blyton, Lucio Miguel Martinez, John McGurk et Peter Turnbull, « Globalisation and Trade Union Strategy : Industrial Restructuring and Human Resource Management in the International Civil Aviation Industry ", article non publié, Cardiff University Department of Industrial Relations, 2000.

44. D. McAdam, « On the International Origins of Domestic Political Opportunities », in Anne Costain et Andrew S. McFarland (dirs.), Social Movements and Political Institutions in the United States, Boulder, Rowman and Littlefield, 1998, chap.15. ; Tarrow Sidney, op.cit., chap. 11.

45. Alberto Melucci, « Getting Involved : Identity and Mobilization in Social Movements », in Bert Klandermans, Hanspeter Kriesi et S. Tarrow (dirs.), From Structure to Action : Comparing Social Movements Across Cultures, International Social Movement Research I, JAI, Greenwich, Connecticut, 1988, pp. 329-348, et A. Melucci, Challenging Codes : Collective Action in the Information Age, Cambridge et New York, Cambridge University Press, 1996 ; David Snow, Burke E. Rochford, Steven Worden et Robert Benford, « Frame Alignment Processes, Micromobilization, and Movement Participation ", American Sociological Review n 51, 1986, pp. 464-481.

46. La révolte des Chiapas a obtenu un soutien international dans la région même mais seulement après que les rebelles eurent acquis suffisamment de notoriété internationale pour que le gouvernement mexicain ne puisse plus se permettre de paraître refuser la présence de militants étrangers.

47. Donatella Della Porta, H. Kriesi et Dieter Rucht (dirs.), Social Movements in a Globalizing World, Londres, MacMillan, 1999 ; M. Keck et K. Sikkink, Activists Beyond Borders, op.cit. ; Jackie Smith, Charles Chatfield et Ron Pagnucco (dirs.), Transnational Social Movements in Global Politics, Syracuse, Syracuse Univerity Press, 1997 ; Boli et Thomas, op.cit.

48. Tom Rochon, Mobilizing for Peace : The Antinuclear Movements in Western Europe, Princeton, Princeton University Press, 1988.

49. Risse, Ropp et Sikkink, op.cit.

50. O. Young (dir.), op.cit.

51. Sanjeev Khagram, «Transnational Struggles for Power and Water : The Political Economy of Big Dam Building and Development in the Third World ", thèse de doctorat non publiée, Stanford University, département de science politique, 1999. S. Khagram, James Riker et K. Sikkink (dirs.), Reconstructing World Politics : Transnational Social Movements and Norms, à paraître. 
52. Alyson Brysk, From Tribal Village to Global Village : Indian Rights and International Relations in Latin America, Irvine, University of California, manuscrit non publié, 1998.

53. Finnemore, op.cit., et Wapner, 1996, op. cit.

54. Smith, Chatfield et Pagnucco, op.cit., et Boli et Thomas, op.cit.

55. M. Keck et K. Sikkink, Activists Beyond Borders, op.cit.

56. Thomas, op.cit.

57. Price, op.cit. 1997.

58. Jeffrey M. Ayres, Defining Conventional Wisdom : Political Movements and Popular Contention against North American Free Trade, Toronto, University of Toronto Press, 1998.

59. Ayres, op.cit. ; Jonathan Fox, « Assessing Binational Civil Society Coalitions ", Chicago/Latino Research Center Working Paper $n^{\circ} 26$, University of California at Santa Cruz, 2000 ; D. Imig et S. Tarrow, « Europeanization of Movements ? Contentious Politics and the European Union, October 1983-March 1995 », in D. della Porta, H. Kriesi et D. Rucht (dirs.), op.cit. ; D. Imig Doug et S. Tarrow Sidney, « Processing Contention in a Europeanising Polity », op.cit. ; D. Imig et S. Tarrow, « Mapping the europeanization of contention : Evidence from a quantitative data analysis », chap.2, in D. Imig et S. Tarrow (dirs.), Contentious Europeans : Protests and Politics in an Integrating Europe, Boulder, Rowman and Littlefield, à paraître.

60. Voir les critiques dans S. Tarrow 1998, op.cit., chap. 11 et Deborah J. Yashar, « Citizenship Claims in Latin America : Parsing out the Role of Globalization », intervention lors de la conférence sur les revendications de citoyenneté, Harvard University Center for International Affairs, octobre 1998.

61. Pour une exception, voir Susanne Rudolf, Hoeber et James Pescatori (dirs.), Transnational Religion and Fading States, Boulder, Westview, 1997.

62. Voir M. Keck Margaret et K. Sikkink, « Social Movements and Transnational Advocacy Networks ", in David S. Meyer et S. Tarrow. (dirs.), Towards a Movement Society? Contentious Politics for a New Century, Boulder, Rowman and Littlefield, 1998 et Tarrow, 1998, op.cit., chap. 11, pour une analyse des similitudes et des différences entre mouvements sociaux et réseaux de militants.

63. Smith, Chatfield et Pagnucco, op.cit.

64. Wapner, 1996, op. cit.

65. Smith, Chatfield et Pagnucco, op.cit., p. 12 et p. 43.

66. Jackie Smith, écrivant en collaboration avec K. Sikkink, semble consciente du problème lorsqu'elle observe : «Les chercheurs ont observé une forte croissance des organisations non-gouvernementales internationales (OING) mais beaucoup d'entre elles ne sont ni des mouvements sociaux ni des réseaux sociaux. » Cf. K. Sikkink et J. Smith, « Infrastructure for Change » in S. Khagram, J. Riker et K. Sikkink (dirs.), Reconstructing World Politics, op. cit.., à comparer avec Smith, Chatfield et Pagnucco, op.cit.

67. M. Keck et K. Sikkink, Activists Beyond Borders, op.cit., p. 210.

68. Mark Anner, « Transnational Campaigns to Defend Labor Rights in Central America's Export Processing Plants », article non publié, School of Industrial and Labor Relations, Cornell University, 1998. Paul Blyton et al, op.cit.

69. Cette définition a été mise au point lors de l'élaboration d'un ouvrage collectif de Doug McAdam, Sidney Tarrow et Charles Tilly, Dynamics of Contention, à paraître. 
70. Cf. C. Tilly, Popular Contention in Great Britain, 1758-1834, op.cit., 1995, Tarrow, 1998, op.cit., et D. McAdam, S. Tarrow et C. Tilly, à paraître, en ce qui concerne la mise au point et l'application de cette définition.

71. Boli et Thomas, op.cit., p. 20

72. les ouvrages de S. Huntington, de M. Keck, K. Sikkink, et de T. Risse, S. Ropp et K. Sikkink emploient le terme mais ne parviennent pas à le définir. Dieter Rucht souligne qu'« on utilise cette étiquette surtout pour désigner toutes les catégories d'acteurs nongouvernementaux, indépendamment des formes qu'ils revêtent, de leur statut formel ou de leurs objectifs ». Cf. D. Rucht, «The Transnationalization of Social Movements : Trends, Causes, Problems » in D. della Porta, H. Kriesi et D. Rucht (dirs.), op.cit., pp. 206-207. Pour Paul Wapner, elles se « définissent le mieux comme des groupes de pression transnationaux qui gagnent en stature politique dans la mesure où ils influencent les actions des Etats ». Cf. Wapner, 1996, op. cit., p. 10. Jackie Smith, Charles Chatfield et Ron Pagnucco se font plus. spécifiques en déclarant que les OING « comportent une large gamme d'organisations ayant des membres issus de pays différents. Elles ont habituellement pour adhérents des associations nationales mais elles incluent souvent également des individus. Elles sont généralement organisées de façon à fournir des services et à promouvoir les intérêts de leurs membres ». Cf. J. Smith, C. Chatfield et R. Pagnucco (dirs.), Transnational Social Movements in Global Politics, op.cit. p. 12. Jackie Smith a ultérieurement ajouté un concept utile à cette définition, «l'autonomie » : les OING « poursuivent des buts indépendamment des gouvernements nationaux et des organismes intergouvernementaux ». Cf. J. Smith, «Transnational Organizations » in Encyclopaedia of Violence, Peace and Conflict, $\mathrm{n}^{\circ} 3$, 1999, p. 591.

73. Boli et Thomas, op.cit., p. 6.

74. Ibid., p. 14.

75. Ibid., p. 19.

76. Ibid., p. 20.

77. A titre d'exemple, les organisations religieuses non-gouvernementales actives en matière de droits de l'homme ou de développement ne sont pas classées comme « religieuses » par l'UAI, ce qui rend difficile la détection du rôle des activités internationales de ces importantes organisations. Je remercie Evelyn Bush d'avoir émis cette observation.

78. D. Meyer et S. Tarrow (dirs.), 1998, op. cit.

79. Boli et Thomas, op.cit., pp. 45-48.

80. Voir la note 77 à titre d'illustration. Le problème que posent les données de l'UAI consiste en général dans l'absence de contrôle systématique de la qualité et de l'exhaustivité des indications fournies par les organisations elles-mêmes et dans le fait que les inférences sur la construction de l'identité proviennent principalement de données sur la croissance numérique des organisations et de leurs militants.

81. D. Meyer et S. Tarrow (dirs.), 1998, op. cit.

82. Boli et Thomas, op.cit., pp. 45-48.

83. M. Keck et K. Sikkink, Activists Beyond Borders, op.cit.

84. M. Keck et K. Sikkink, « Social Movements and Transnational Advocacy Networks ", in David S. Meyer et Sidney Tarrow (dirs.), Towards a Movement Society ? Contentious Politics for a New Century, op.cit. ] ? 婜 La grande majorité des travaux empiriques concernant les réseaux transnationaux de militants ayant porté sur des groupes défendant des « normes », la même logique de formation d'une coalition et de 
déploiement du pouvoir de pays tiers et/ou d'institutions internationales prévaut-elle lorsque le mouvement se fonde sur un intérêt matériel? 賱 Les réseaux transnationaux de militants jouent-ils un rôle d'intrus occasionnel entre les Etats et leurs ressortissants ou servent-ils de liens fondamentaux dans la formation de mouvements sociaux transnationaux entre citoyens de différents pays? 盛 Enfin, quelles sont les relations que les réseaux transnationaux de militants entretiennent avec les institutions internationales? Dans le paradigme de M. Keck et K. Sikkink, les institutions servent de liens intermédiaires entre les réseaux de militants et leurs alliés. Dans la mesure où les militants dépendent de ces institutions, dans quelle mesure leurs campagnes peuventelles aller plus loin que les lignes politiques de ces dernières ? Cette question devient essentielle dans le cas de l'Union européenne. Institutions internationales et contestation transnationale L'étude de la contestation transnationale se divise en deux courants principaux, la thèse de la « société civile mondiale » dont les adeptes tendent à souligner la place du conflit et un degré inexorablement croissant de contestation transnationale, et une perspective institutionnaliste prenant en compte tant le conflit que la coopération et prévoyant un degré variable d'activité transnationale. La thèse de la " société civile mondiale », bien connue, peut donc se résumer rapidement: 䀡 A l'ère de la diffusion à l'échelon mondial des programmes de télévision, de l'Internet et du courrier électronique, les structures d'opportunités politiques à l'échelon national peuvent être remplacées par leurs équivalents transnationaux pour monter des actions collectives. 瞘 L'Etat-nation est peut-être en train de perdre sa capacité à circonscrire et à structurer l'action collective. Cela provient en partie de l'incapacité croissante des gouvernements à dissimuler à leurs ressortissants les événements ayant lieu à l'étranger. Mais cela est également dû au fait que l'intégration de l'économie internationale affaiblit la capacité des Etats à faire face aux tendances économiques mondiales. 臨 Cette incapacité croissante des Etats à contrôler l'économie mondiale a permis à des individus et à des groupes d'avoir accès à de nouvelles sortes de ressources pour organiser des actions collectives par delà les frontières. Il s'agit notamment des voyages à l'étranger, des contacts avec des personnes partageant la même opinion dans d'autres pays et d'un savoir-faire croissant dans le maniement de la communication transnationale Enfin, dans un contexte de mondialisation de l'économie, d'universalisation des cultures et de prolifération des institutions, les idéaux sont de plus en plus adoptés comme des normes internationales[[M. Finnemore, op.cit.

85. S. Tarrow 1998, op.cit., chap. 11.

86. D. Imig et S. Tarrow, « Processing Contention in a Europeanising Polity », op.cit. à paraître.

87. T. Risse-Kappen, 1995, op.cit..

88. T. Risse, 2000, op.cit., p. 27.

89. R. O. Keohane, International Institutions and State Power, op.cit.

90. M. Finnemore et K. Sikkink, «International Norm Dynamics and Political Change », op.cit., pp. 887-917.

91. Cette partie résume les conclusions de l'ouvrage dirigé par D. Imig et S. Tarrow, Contentious Europeans : Protest and Politics in an Integrating Europe, op. cit.

92. Ibid..

93. A. Moravcsik, op.cit., 1998.

94. Boli et Thomas, op.cit., pp. 28-30. 
INDEX

Mots-clés : Mouvements sociaux, résistances politiques, réseaux transnationaux, mobilisation

\section{AUTEUR}

\section{SIDNEY TARROW}

J'ai rédigé cet article alors que j'étais membre du Forum Européen du Centre Robert Schuman de l'Institut universitaire européen. J'en ai présenté des versions lors de la réunion annuelle de 1999 de l'American Sociological Association et lors de l'atelier sur la politique de la contestation à l'Université de Columbia. Je tiens à remercier Tanja Boerzel, John Boli, Matt Evangelista, Doug Imig, Christian Joppke, Tom Loya, Roger Karapin, Peter Katzenstein, Hanspeter Kriesi, Doug McAdam, John Meyer, Craig Murphy, Thomas Risse, Jackie Smith, Charles Tilly et mes confrères Fellows à la Villa la Fonte pour avoir lu et commenté mes versions antérieures en proposant des idées et des critiques qui m'ont été précieuses. 